\title{
О КЛАССИЧЕСКОМ РЕШЕНИИ СМЕШАННОЙ ЗАДАЧИ ДЛЯ ЛИНЕЙНОГО НЕСТРОГО ГИПЕРБОЛИЧЕСКОГО УРАВНЕНИЯ ЧЕТВЕРТОГО ПОРЯДКА С ОДНОЙ КРАТНОЙ ХАРАКТЕРИСТИКОЙ
}

\author{
Е. С. Чеб, Е. С. Симинская \\ Статъя представлена членом редакционной коллегии А. П. Солдатовым \\ Белорусский государственный университет \\ г. Минск, 220030, Республика Беларусь \\ E-mail: cheb@bsu.by, Slavelena16@gmail.com
}

\begin{abstract}
Аннотация. Данная статья посвящена построению классического решения смешанной задачи для линейного однородного нестрого гиперболического уравнения четвертого порядка, оператор которого представляет собой четырехкратную композицию одного и того же оператора первого порядка, с постоянными коэффициентами и четырехкратной характеристикой. Для корректной постановки данной задачи граничные условия задаются не на всей боковой границе, что является ее особенностью. Для построения решения используется метод характеристик. Согласно этому методу, в общем решении исходного уравнения присутствуют четыре неизвестные функции, которые на области определения находятся из начальных и граничных условий. Классическое решение построено для случая отсутствия производных младших порядков. Получены достаточные условия гладкости и согласований граничных условий с начальными условиями и уравнением. Гладкость исходных данных нужна для четырежды непрерывной дифференцируемости решения. Условия согласования нужны для четырежды непрерывной дифференцируемости решения на критической характеристике. Доказана теорема существования единственного классического решения этой смешанной задачи. Полученные результаты могут быть применены в теории уравнений с частными производными и в вычислительной математике.
\end{abstract}

Ключевые слова: нестрого гиперболическое уравнение четвертого порядка, смешанная задача, классическое решение, метод характеристик, условия гладкости и согласования.

Для цитирования: Чеб Е. С., Симинская Е. С. 2020. О классическом решении смешанной задачи для линейного нестрого гиперболического уравнения четвертого порядка с одной кратной характеристикой. Прикладная математика \& Физика. 52(1): 11-17. DOI 10.18413/2687-0959-2020-52-1-11-17.

\section{CLASSICAL SOLUTION OF THE MIXED PROBLEM FOR LINEAR NONSTRICTLY HYPERBOLIC FOURTH-ORDER WITH MULTIPLE CHATACTERISTIC}

\author{
E. S. Cheb, E. S. Siminskaya \\ Article submitted by a member of the editorial board A. P. Soldatov \\ Belorussian State University, \\ Minsk, 220030, Republic of Belarus \\ E-mail: cheb@bsu.by, Slavelena16@gmail.com \\ Received January 10, 2020
}

\begin{abstract}
In the work is constructed the classical solution of mixed value problem for not strictly hyperbolic homogeneous equation of the fourth order with constant coefficients and the multiple characteristics. We use the method of characteristics to solve this problem. According to this method, general solution of the equation contains the sum of four functions, which are found from initial and boundary conditions. This general solution is constructed for the both cases: with the presence or absence derivatives of lower orders. We obtained matching conditions for initial and boundary conditions. These conditions follow from the requirement of four times continuous differentiability of the solution taking into account smoothness of the functions. A theorem on the existence of a unique classical solution has been proved. The obtained results can be used in the theory of differential equations with partial derivatives and in the computational mathematics.

Key words: partial differential hyperbolic equation of the fourth order, initial value problem, boundary value problem, classical solution, method of characteristics, smoothness and matching conditions.

For citation: Cheb E. S., Siminskaya E. S. 2020. Classical Solution of the Mixed Problem for Linear Nonstrictly Hyperbolic Fourth-Order with Multiple Characteristic. Applied Mathematics \& Physics. 52(1): 11-17 (in Russian). DOI 10.18413/2687-0959-2020-52-1-11-17.
\end{abstract}


Введение. Нестрого гиперболические уравнения обладают рядом особенностей, которые появляются при постановке и исследовании на разрешимость смешанных задач для них. Среди них наиболее изученной является задача Коши. В работе [Иврий, Петков, 1974] получены необходимые условия корректности задачи Коши для нестрого гиперболических уравнений второго порядка. Некоторые смешанные задачи для уравнений гиперболического типа были исследованы [Sakamoto, 1970]. На возможность применения метода характеристик при решении смешанных задач указано в работе [Than, Mikio, Nguen, 2000]. В работах [Ломовцев, Юрчук, 2016] и [Ломовцев, 2019] методом характеристик получены необходимые и достаточные условия корректной разрешимости смешанных задач для нестрого гиперболического уравнения второго порядка с двухкратной характеристикой соответственно в первой четверти и полуполосе плоскости. В этих работах указано на повышение требований гладкости от граничных с начальными данными и уравнением. В [Чеб, 2017] методом характеристик получено классическое решение смешанной задачи для нестрого гиперболического уравнения четвертого порядка, оператор которого представляет собой композицию двух операторов второго порядка.

В данной работе впервые рассматривается смешанная задача для уравнения четвертого порядка с одной характеристикой кратности четыре. Особенность изучаемой задачи заключается в том, что граничные условия задаются не на всей боковой границе.

1. Постановка задачи. В области $Q=(0, \infty) \times \Omega \subset \mathbb{R}^{2}, \mathbb{R}=(-\infty,+\infty)$, переменных $(t, x)$ рассмотрим относительно функции $u(t, x)$ линейное нестрого гиперболическое уравнение четвертого порядка с постоянными коэффициентами в предположении, что гиперболический оператор уравнения $L$ представим в виде композиции операторов первого порядка

$$
L u \equiv \prod_{i=1}^{4}\left(\frac{\partial}{\partial t}-a \frac{\partial}{\partial x}\right) u(t, x)=0, \quad a>0, \quad(t, x) \in Q, \quad \text { где } \quad \Omega=(0, l), l>0
$$

K уравнению (1) присоединим начальные условия

$$
\left.\frac{\partial^{j} u}{\partial t^{j}}\right|_{t=0}=\varphi_{j}(x), \quad x \in \Omega, \quad j=0,1,2,3
$$

и граничные условия

$$
\begin{gathered}
u(t, 0)=\mu_{1}(t),\left.\quad \frac{\partial u}{\partial x}\right|_{x=0}=\mu_{2}(t), \quad t \in\left(\frac{l}{a},+\infty\right), \\
u(t, l)=\nu_{1}(t),\left.\quad \frac{\partial u}{\partial x}\right|_{x=l}=\nu_{2}(t), \quad t \in(0,+\infty) .
\end{gathered}
$$

Условия (3)-(4) выбираются таким образом, чтобы смешанная (начально-граничная) задача (1)(4) была корректно поставленной по Адамару, т. е. всегда существовало единственное и устойчивое по исходным данным ее классическое решение. Требуется построить классическое решение $u \in C^{(4)}(\bar{Q}), \bar{Q}=[0, \infty) \times[0, l]$, уравнения (1) из класса четырежды непрерывно дифференцируемых функций, удовлетворяющих уравнению (1) на $Q$ в обычном смысле, а начальным (2) и граничным условиям (3)-(4) в смысле пределов значения решения $u(t, x)$ во внутренних точках $(t, x) \in Q$. Например, для начального условия (2) при $j=0: \forall x \in \Omega, \forall\left(t_{n}, x_{n}\right) \in Q,\left(t_{n}, x_{n}\right) \rightarrow(0, x)$ в $\mathbb{R}^{2}$ при $n \rightarrow \infty$ последовательность $u\left(t_{n}, x_{n}\right) \rightarrow \varphi_{0}(x)$ в $\mathbb{R}$ при $n \rightarrow \infty$. Найти достаточные требования гладкости исходных данных $\varphi_{j}(j=\overline{0,3}), \mu_{1}, \mu_{2}, \nu_{1}, \nu_{2}$ и условия согласования между ними и уравнением для корректной разрешимости поставленной задачи (1)-(4).

2. Решение смешанной задачи. Уравнение (1) имеет одно семейство характеристик $x+a t=$ $C, C \in \mathbb{R}$, кратности четыре. Область $Q$ разбивается характеристикой $x+a t=l$ на две подобласти

$$
Q^{(0)}=\left\{(t, x): 0<t<\frac{l-x}{a}, 0<x<l\right\}, Q^{(1)}=\left\{(t, x): t>\frac{l-x}{a}, 0<x<l\right\},
$$

$\bar{Q}=\overline{Q^{(0)}} \cup \overline{Q^{(1)}}$. Эту характеристику $x+a t=l$ будем называть критической по аналогии с работой [Ломовцев, Юрчук, 2016].

Из определения классического решения $u \in C^{(4)}(\bar{Q})$ задачи (1)-(4) вытекают основные обязательные требования гладкости начальных и граничных данных

$$
\begin{array}{r}
\varphi_{0} \in C^{(4)}(\bar{\Omega}), \varphi_{1} \in C^{(3)}(\bar{\Omega}), \varphi_{2} \in C^{(2)}(\bar{\Omega}), \varphi_{3} \in C^{(1)}(\bar{\Omega}), \\
\mu_{1} \in C^{(4)}\left[\frac{l}{a}, \infty\right), \mu_{2} \in C^{(3)}\left[\frac{l}{a}, \infty\right), \nu_{1} \in C^{(4)}[0, \infty), \nu_{2} \in C^{(3)}[0, \infty) .
\end{array}
$$


Вычисляя соответствующие производные по $t$ от граничных условий (4) при $t=0$, по $x$ из начальных условий (2) при $x=0$, получаем простейшие условия согласования граничных условий (4) с начальными условиями (2) и уравнением (1):

$$
\begin{gathered}
\left.\frac{d^{s} \nu_{1}(t)}{d t^{s}}\right|_{t=0}=\varphi_{s}(l),\left.\quad \frac{d^{s} \nu_{2}(t)}{d t^{s}}\right|_{t=0}=\varphi^{\prime}{ }_{s}(l), s=0,1,2,3 \\
\nu_{1}^{(4)}(0)=4 a \varphi_{3}^{\prime}(l)-6 a^{2} \varphi_{2}^{\prime \prime}(l)+4 a^{3} \varphi_{1}^{(3)}(l)-a^{4} \varphi_{0}^{(4)}(l) .
\end{gathered}
$$

Условие согласования (7) вытекает из уравнения (1), начальных условий (2) и первого граничного условия из (4). Остальные достаточные условия согласования будут получены в процессе доказательства следующей теоремы.

Теорема. Пусть выполнены требования гладкости началъных и граничных данных $\varphi_{0} \in C^{(7)}(\bar{\Omega})$, $\varphi_{1} \in C^{(6)}(\bar{\Omega}), \varphi_{2} \in C^{(5)}(\bar{\Omega}), \varphi_{3} \in C^{(4)}(\bar{\Omega}), \mu_{1} \in C^{(5)}\left[\frac{l}{a}, \infty\right), \nu_{1} \in C^{(5)}[0, \infty), \mu_{2} \in C^{(4)}\left[\frac{l}{a}, \infty\right)$, $\nu_{2} \in C^{(4)}[0, \infty)$, и выполнены основные условия согласования (6), (7) и дополнительные условия согласования

$$
\begin{aligned}
& \mu_{i+1}\left(\frac{l}{a}\right)=\frac{1}{6 a^{3}}\left[6 a^{3} \varphi_{0}^{(i)}(l)+6 a^{2} l \varphi_{1}^{(i)}(l)+3 a l^{2} \varphi_{2}^{(i)}(l)+l^{3} \varphi_{3}^{(i)}(l)-6 a^{3} \varphi_{0}^{(i+1)}(l)\right]+ \\
& +\frac{1}{6 a^{3}}\left[-6 a^{2} l^{2} \varphi_{1}^{(i+1)}(l)-3 a l^{3} \varphi_{2}^{(i+1)}(l)+3 a^{3} l^{2} \varphi_{0}^{(i+2)}(l)+3 a^{2} l^{3} \varphi_{1}^{(i+2)}(l)-a^{3} l^{3} \varphi_{0}^{(i+3)}(l)\right] \\
& \mu_{i+1}^{\prime}\left(\frac{l}{a}\right)=\frac{1}{6 a^{2}}\left[6 a^{2} \varphi_{1}^{(i)}(l)+6 a l \varphi_{2}^{(i)}(l)+3 l^{2} \varphi_{3}^{(i)}(l)\right]+ \\
& +\frac{1}{6 a^{2}}\left[-6 a^{2} l \varphi_{1}^{(i+1)}(l)-6 a l^{2} \varphi_{2}^{(i+1)}(l)+l^{3} \varphi_{3}^{(i+1)}(l)\right]+ \\
& +\frac{1}{6 a^{2}}\left[3 a^{2} l^{2} \varphi_{1}^{(i+2)}(l)-3 a l^{3} \varphi_{2}^{(i+2)}(l)+3 a^{2} l^{3} \varphi_{1}^{(i+3)}(l)-a^{3} l^{3} \varphi_{0}^{(i+4)}(l)\right], \\
& \mu_{i+1}^{\prime \prime}\left(\frac{l}{a}\right)=\frac{1}{6 a}\left[6 a \varphi_{2}^{(i)}(l)+6 l \varphi_{3}^{(i)}(l)-6 a l \varphi_{2}^{(i+1)}(l)+6 l^{2} \varphi_{3}^{(i+1)}(l)-15 a l^{2} \varphi_{2}^{(i+2)}(l)\right]+ \\
& +\frac{1}{6 a}\left[l^{3} \varphi_{3}^{(i+2)}(l)+12 a^{2} l^{2} \varphi_{1}^{(i+3)}(l)\right]+ \\
& +\frac{1}{6 a}\left[-3 a l^{3} \varphi_{2}^{(i+3)}(l)-3 a^{3} l^{2} \varphi_{0}^{(i+4)}(l)+3 a^{2} l^{3} \varphi_{1}^{(i+4)}(l)-a^{3} l^{3} \varphi_{0}^{(i+5)}(l)\right], \\
& \mu_{i+1}^{(3)}\left(\frac{l}{a}\right)=\frac{1}{6}\left[6 \varphi_{3}^{(i)}(l)+18 l \varphi_{3}^{(i+1)}(l)-36 a l \varphi_{2}^{(i+2)}(l)+9 l^{2} \varphi_{3}^{(i+2)}(l)+24 a^{2} l \varphi_{1}^{(i+3)}(l)\right]+ \\
& +\frac{1}{6}\left[-24 a l^{2} \varphi_{2}^{(i+3)}(l)+l^{3} \varphi_{3}^{(i+3)}(l)-6 a^{3} l \varphi_{0}^{(i+4)}(l)+21 a^{2} l^{2} \varphi_{1}^{(i+4)}(l)-3 a l^{3} \varphi_{2}^{(i+4)}(l)\right]+ \\
& +\frac{1}{6}\left[-6 a^{3} l^{2} \varphi_{0}^{(i+5)}(l)+3 a^{2} l^{3} \varphi_{1}^{(i+5)}(l)-a^{3} l^{3} \varphi_{0}^{(i+6)}(l)\right], i=0,1, \\
& \mu_{1}^{(4)}\left(\frac{l}{a}\right)=\frac{a}{6}\left[24 \varphi_{3}^{\prime}(l)-36 a \varphi_{2}^{\prime \prime}(l)+36 l \varphi_{3}^{\prime \prime}(l)+24 a^{2} \varphi_{1}^{(3)}(l)-84 a l \varphi_{2}^{(3)}(l)+12 l^{2} \varphi_{3}^{(3)}(l)\right]+ \\
& +\frac{a}{6}\left[-6 a^{3} \varphi_{0}^{(4)}(l)+66 a^{2} l \varphi_{1}^{(4)}(l)-33 a l^{2} \varphi_{2}^{(4)}(l)+l^{3} \varphi_{3}^{(4)}(l)-18 a^{3} l \varphi_{0}^{(5)}(l)\right]+ \\
& +\frac{a}{6}\left[30 a^{2} l^{2} \varphi_{1}^{(5)}(l)-3 a l \varphi_{2}^{(5)}(l)-9 a^{3} l^{2} \varphi_{0}^{(6)}(l)+3 a^{2} l^{3} \varphi_{1}^{(6)}(l)-a^{3} l^{3} \varphi_{0}^{(7)}(l)\right], \\
& \mu_{2}^{(4)}\left(\frac{l}{a}\right)-\frac{1}{a} \mu_{1}^{(5)}\left(\frac{l}{a}\right)=\frac{a}{2}\left[-12 \varphi_{3}^{\prime \prime}(l)+28 a \varphi_{2}^{(3)}(l)-8 l \varphi_{3}^{(3)}(l)-22 a^{2} \varphi_{1}^{(4)}(l)\right]+ \\
& +\frac{a}{2}\left[22 a l \varphi_{2}^{(4)}(l)-l^{2} \varphi_{3}^{(4)}(l)+6 a^{3} \varphi_{0}^{(5)}(l)-20 a^{2} l \varphi_{1}^{(5)}(l)+3 a l \varphi_{2}^{(5)}(l)\right]+ \\
& +\frac{a}{2}\left[6 a^{3} l \varphi_{0}^{(6)}(l)-3 a^{2} l^{2} \varphi_{1}^{(6)}(l)+a^{3} l^{2} \varphi_{0}^{(7)}(l)\right] \\
& \nu_{1}^{(5)}(0)-a \nu_{2}^{(4)}(0)=6 a^{2} \varphi_{3}^{\prime \prime}(l)-12 a^{3} \varphi_{2}^{(3)}(l)+11 a^{4} \varphi_{1}^{(4)}(l)-3 a^{5} \varphi_{0}^{(5)}(l) \text {. }
\end{aligned}
$$

Тогда в классе функций $C^{(4)}(\bar{Q})$ смешанная задача (1)-(4) имеет единственное классическое решение

$$
\begin{aligned}
& u_{0}(t, x)=\varphi_{0}(x+a t)+t\left[\varphi_{1}(x+a t)-a \varphi_{0}^{\prime}(x+a t)\right]+\frac{1}{2} t^{2}\left[\varphi_{2}(x+a t)-2 a \varphi_{1}^{\prime}(x+a t)+\right. \\
& \left.+a^{2} \varphi_{0}^{\prime \prime}(x+a t)\right]+\frac{1}{6} t^{3}\left[\varphi_{3}(x+a t)-3 a \varphi_{2}^{\prime}(x+a t)+3 a^{2} \varphi_{1}^{\prime \prime}(x+a t)-a^{3} \varphi_{0}^{(3)}(x+a t)\right],(t, x) \in Q^{(0)}
\end{aligned}
$$




$$
\begin{array}{r}
u_{1}(t, x)=\frac{(l-x)^{2}(l+2 x)}{l^{3}} \mu_{1}\left(t+\frac{l}{a}\right)+\frac{x(l-x)^{2}}{l^{2}} \mu_{2}\left(t+\frac{l}{a}\right)+ \\
+\frac{x^{2}(3 l-2 x)}{l^{3}} \nu_{1}\left(t-\frac{l-x}{a}\right)-\frac{x^{2}(l-x)}{l^{2}} \nu_{2}\left(t-\frac{l-x}{a}\right)- \\
-\frac{x(l-x)^{2}}{a l^{2}} \mu_{1}^{\prime}\left(t+\frac{l}{a}\right)+\frac{x^{2}(l-x)}{a l^{2}} \nu_{1}^{\prime}\left(t-\frac{l-x}{a}\right),(t, x) \in Q^{(1)} .
\end{array}
$$

Доказательство. Методом характеристик найдем общий вид классических решений уравнения (1) на $Q$.

Лемма. Общее решение уравнения (1) на $Q$ из класса $C^{(4)}(Q)$ четырежды непрерывно дифферениируемых функиий представляется в виде суммы

$$
u(t, x)=g_{1}(x+a t)+t g_{2}(x+a t)+t^{2} g_{3}(x+a t)+t^{3} g_{4}(x+a t)
$$

четырех произвольных функиий $g_{1}, g_{2}, g_{3}, g_{4} \in C^{(4)}(\mathbb{R})$ от аргумента $x+$ at, где функции $g_{i}:$ $[0, \infty) \ni y \rightarrow g_{i}(y) \in \mathbb{R}, i=1,2,3,4$.

Доказательство леммы. Сделаем замену переменных $\xi=x+a t, \eta=t$ в уравнении (1) и приведем его к каноническому виду $\frac{\partial^{4} \widetilde{u}}{\partial \eta^{4}}=0$, где $\widetilde{u}(\eta, \xi)=u(t, x)$. Интегрируя последнее уравнение четырежды по переменной $\eta$ при каждом фиксированном $\xi$, находим $\widetilde{u}(\eta, \xi)=g_{1}(\xi)+\eta g_{2}(\xi)+$ $\eta^{2} g_{3}(\xi)+\eta^{3} g_{4}(\xi)$. Возвращаясь здесь к старым переменным, получаем общее решение (11), так как сделанная замена переменных не вырождена. Лемма доказана.

Из леммы следует, что для того, чтобы решить задачу (1)-(4), нам нужно определить функции $g_{i}, i=1,2,3,4$, так, чтобы выполнялись условия (2)-(4). Классическое решение задачи (1)-(4) в области $Q^{(0)}$ совпадает с классическим решением задачи Коши $(1),(2)$ в $Q^{(0)}$. Найдем ее решение. Удовлетворяя (11) начальным условиям (2) в указанном выше смысле, получаем систему уравнений

$$
\begin{aligned}
& g_{1}(x)=\varphi_{0}(x), \quad g_{2}(x)+a g_{1}^{\prime}(x)=\varphi_{1}(x), \quad 2 g_{3}(x)+2 a g_{2}^{\prime}(x)+a^{2} g_{1}^{\prime \prime}(x)=\varphi_{2}(x), \\
& 6 g_{4}(x)+6 a g_{3}^{\prime}(x)+3 a^{2} g_{2}^{\prime \prime}(x)+a^{3} g_{1}^{(3)}(x)=\varphi_{3}(x), x \in(0, l) .
\end{aligned}
$$

Система (12) решается стандартными методами, а именно, из первого уравнения выражаем $g_{1}$, затем дифференцируем первое уравнение и подставляем $g_{1}^{\prime}$ во второе, из которого выражаем $g_{2}$ и т. д. На каждом шаге получается алгебраическое уравнение. Таким образом, из системы (12) однозначно определяются функции $g_{1}, g_{2}, g_{3}, g_{4}$, когда их аргумент $y \in(0, l)$. Обозначим их через $g_{1}^{(0)}, g_{2}^{(0)}, g_{3}^{(0)}, g_{4}^{(0)}$. Тогда

$$
\begin{gathered}
g_{1}^{(0)}(y)=\varphi_{0}(y) \\
g_{2}^{(0)}(y)=\varphi_{1}(y)-a \varphi_{0}^{\prime}(y) \\
g_{3}^{(0)}(y)=\frac{1}{2}\left[\varphi_{2}(y)-2 a \varphi_{1}^{\prime}(y)+a^{2} \varphi_{0}^{\prime \prime}(y)\right] \\
g_{4}^{(0)}(y)=\frac{1}{6}\left[\varphi_{3}(y)-3 a \varphi_{2}^{\prime}(y)+3 a^{2} \varphi_{1}^{\prime \prime}(y)-a^{3} \varphi^{(3)}(y)\right] .
\end{gathered}
$$

Следовательно, в представлении (11) по формулам (13)-(16) мы однозначно определили $g_{i}(y),(i=$ $\overline{1,4})$, когда $y \in(0, l)$, и тем самым решили задачу Коши (1), (2). Ее единственным формальным решением является функция

$$
u_{0}(t, x)=g_{1}^{(0)}(x+a t)+t g_{2}^{(0)}(x+a t)+t^{2} g_{3}^{(0)}(x+a t)+t^{3} g_{4}^{(0)}(x+a t),
$$

которая при подстановке в нее выражений $(13)-(16)$ для $g_{i}^{(0)}(y)(i=\overline{1,4})$ совпадает с $(9)$.

Поскольку надо, чтобы $u_{0} \in C^{(4)}\left(\overline{Q^{(0)}}\right)$, то достаточные требования на гладкость начальных функций имеют вид

$$
\varphi_{0} \in C^{(7)}(\bar{\Omega}), \varphi_{1} \in C^{(6)}(\bar{\Omega}), \varphi_{2} \in C^{(5)}(\bar{\Omega}), \varphi_{3} \in C^{(4)}(\bar{\Omega})
$$

Как видно, условия (18) отличаются от условий (5). Повышение требований гладкости обусловлено тем, что уравнение (1) является нестрого гиперболическим. Такая необходимость повышения гладкости была подмечена при решении смешанной задачи для нестрого гиперболического уравнения второго порядка в [Ломовцев, Юрчук, 2016].

Как видно из алгоритма вывода формулы (17), задача Коши имеет единственное решение. Решив задачу Коши, мы нашли классическое решение исходной задачи в области $Q^{(0)}$. Будем искать 
теперь классическое решение задачи в $Q^{(1)}$. Для определения функций $g_{i}(y)(i=\overline{1,4})$ на оставшейся области определения $\overline{Q^{(1)}}$ воспользуемся граничными условиями $(3)-(4)$.

На концах $x=0$ и $x=l$ задаются граничные условия по два на каждом, можно найти поэтому значения функций $g_{i}(y)(i=\overline{1,4})$. Сначала рассмотрим левую границу $x=0$. Подставим в $(11)$ и в его первую производную по $x$ значение $x=0$ и получим систему вида

$$
\begin{aligned}
& g_{1}(a t)+t g_{2}(a t)+t^{2} g_{3}(a t)+t^{3} g_{4}(a t)=\mu_{1}(t), \\
& g_{2}^{\prime}(a t)+t g_{2}^{\prime}(a t)+t^{2} g_{3}^{\prime}(a t)+t^{3} g_{4}^{\prime}(a t)=\mu_{2}(t) .
\end{aligned}
$$

В силу того, что $t>l / a$, то аргумент at $>l$, поэтому система (19) содержит четыре неизвестные функции. Преобразуем уравнения в (19). Сделаем замену $y=a t$, первое уравнение продифференцируем по переменной $y$ и вычтем из полученного второе уравнение системы. Система (19) примет вид

$$
\begin{gathered}
g_{1}(y)+\frac{y}{a} g_{2}(y)+\left(\frac{y}{a}\right)^{2} g_{3}(y)+\left(\frac{y}{a}\right)^{3} g_{4}(y)=\mu_{1}\left(\frac{y}{a}\right), \\
g_{2}(y)+2 \frac{y}{a} g_{3}(y)+3\left(\frac{y}{a}\right)^{2} g_{4}(y)=a \mu_{1}^{\prime}\left(\frac{y}{a}\right)-a \mu_{2}\left(\frac{y}{a}\right) .
\end{gathered}
$$

Перейдем к рассмотрению условий на правой границе $x=l$. Удовлетворив (11) условиям (4), получим

$$
\begin{aligned}
& g_{1}(l+a t)+t g_{2}(l+a t)+t^{2} g_{3}(l+a t)+t^{3} g_{4}(l+a t)=\nu_{1}(t), \\
& g_{2}^{\prime}(l+a t)+t g_{2}^{\prime}(l+a t)+t^{2} g_{3}^{\prime}(l+a t)+t^{3} g_{4}^{\prime}(l+a t)=\nu_{2}(t) .
\end{aligned}
$$

Аналогично, как и в системе (19), сделаем замену $y=l+a t$, тогда (21) примет вид

$$
\begin{gathered}
g_{1}(y)+\frac{y-l}{a} g_{2}(y)+\left(\frac{y-l}{a}\right)^{2} g_{3}(y)+\left(\frac{y-l}{a}\right)^{3} g_{4}(y)=\nu_{1}\left(\frac{y-l}{a}\right), \\
g_{2}(y)+2 \frac{y-l}{a} g_{3}(y)+3\left(\frac{y-l}{a}\right)^{2} g_{4}(y)=a \nu_{1}^{\prime}\left(\frac{y-l}{a}\right)-a \nu_{2}\left(\frac{y-l}{a}\right) .
\end{gathered}
$$

Таким образом, для определения функций $g_{i}(y)(i=\overline{1,4})$ для $y \in(l, \infty)$, мы получили систему четырех уравнений $(20),(22)$ с четырьмя неизвестными, решая которую определяем эти функции. Обозначим их через $g_{1}^{(1)}, g_{2}^{(1)}, g_{3}^{(1)}, g_{4}^{(1)}$. Тогда

$$
\begin{gathered}
g_{1}^{(1)}(y)=\frac{(l-y)^{2}(l+2 y)}{l^{3}} \mu_{1}\left(\frac{y}{a}\right)+\frac{y(l-y)^{2}}{l^{2}} \mu_{2}\left(\frac{y}{a}\right)+\frac{y^{2}(3 l-2 y)}{l^{3}} \nu_{1}\left(\frac{y-l}{a}\right)- \\
-\frac{y^{2}(l-y)}{l^{2}} \nu_{2}\left(\frac{y-l}{a}\right)-\frac{y(l-y)^{2}}{a l^{2}} \mu_{1}^{\prime}\left(\frac{y}{a}\right)+\frac{y^{2}(l-y)}{a l^{2}} \nu_{1}^{\prime}\left(\frac{y-l}{a}\right), \\
g_{2}^{(1)}(y)=\frac{6 a y(l-y)}{l^{3}} \mu_{1}\left(\frac{y}{a}\right)-\frac{a(l-3 y)(l-y)}{l^{2}} \mu_{2}\left(\frac{y}{a}\right)-\frac{6 a y(l-y)}{l^{3}} \nu_{1}\left(\frac{y-l}{a}\right)+ \\
+\frac{a y(2 l-3 y)}{l^{2}} \nu_{2}\left(\frac{y-l}{a}\right)+\frac{(l-3 y)(l-y)}{l^{2}} \mu_{1}^{\prime}\left(\frac{y}{a}\right)-\frac{y(2 l-3 y)}{l^{2}} \nu_{1}^{\prime}\left(\frac{y-l}{a}\right), \\
g_{3}^{(1)}(y)=-\frac{3(l-2 y)}{l^{3}} \mu_{1}\left(\frac{y}{a}\right)-\frac{a^{2}(2 l-3 y)}{l^{2}} \mu_{2}\left(\frac{y}{a}\right)+\frac{3 a^{2}(l-2 y)}{l^{3}} \nu_{1}\left(\frac{y-l}{a}\right)- \\
-\frac{a^{2}(l-3 y)}{l^{2}} \nu_{2}\left(\frac{y-l}{a}\right)+\frac{a(2 l-3 y)}{l^{2}} \mu_{1}^{\prime}\left(\frac{y}{a}\right)+\frac{a(l-3 y)}{l^{2}} \nu_{1}^{\prime}\left(\frac{y-l}{a}\right), \\
g_{4}^{(1)}(y)=-\frac{2 a^{3}}{l^{3}} \mu_{1}\left(\frac{y}{a}\right)-\frac{a^{3}}{l^{2}} \mu_{2}\left(\frac{y}{a}\right)+\frac{2 a^{3}}{l^{3}} \nu_{1}\left(\frac{y-l}{a}\right)- \\
-\frac{a^{3}}{l^{2}} \nu_{2}\left(\frac{y-l}{a}\right)+\frac{a^{2}}{l^{2}} \mu_{1}^{\prime}\left(\frac{y}{a}\right)+\frac{a^{2}}{l^{2}} \nu_{1}^{\prime}\left(\frac{y-l}{a}\right) .
\end{gathered}
$$

Из формул (23)-(26) видно, что функции $g_{i}^{(1)}(i=\overline{1,4})$ в представлении (11) определяются единственным образом, поэтому формальное решение исходной задачи в области $Q^{(1)}$ определяется однозначно и имеет вид

$$
u_{1}(t, x)=g_{1}^{(1)}(x+a t)+t g_{2}^{(1)}(x+a t)+t^{2} g_{3}^{(1)}(x+a t)+t^{3} g_{4}^{(1)}(x+a t),
$$

где $g_{i}^{(1)}, i=\overline{1,4}$, находятся по формулам (23)-(26). Подставив их, после преобразований получим формулу (10). Из-за нестрогой гиперболичности уравнения (1) для того, чтобы $u_{1} \in C^{(4)}\left(\overline{Q^{(1)}}\right)$ достаточно гладкости граничных данных $\mu_{i}, \nu_{1}$ на единицу больше, чем указано в (5). Из вывода формулы (27) вытекает единственность решения (10) этой граничной задачи. 
Решение $u(t, x)$ в области $Q$ определяется двумя формулами: $u_{0}(t, x)$, если $(t, x) \in Q^{(0)}$, и $u_{1}(t, x)$, если $(t, x) \in Q^{(1)}$. Теперь нужно показать, что на характеристике $x+a t=l$ решения (9) и (10) непрерывны и имеют непрерывные производные до четвертого порядка включительно, т. е. выполняются следующие соотношения:

$$
\begin{aligned}
& u_{0}(t, l-a t)=u_{1}(t, l-a t),\left.\frac{\partial^{s} u_{0}}{\partial t^{s}}\right|_{x=l-a t}=\left.\frac{\partial^{s} u_{1}}{\partial t^{s}}\right|_{x=l-a t},\left.\frac{\partial^{s} u_{0}}{\partial x^{s}}\right|_{x=l-a t}=\left.\frac{\partial^{s} u_{1}}{\partial x^{s}}\right|_{x=l-a t}, s=1,2,3,4, \\
& \left.\frac{\partial^{2} u_{0}}{\partial t \partial x}\right|_{x=l-a t}=\left.\frac{\partial^{2} u_{1}}{\partial t \partial x}\right|_{x=l-a t},\left.\frac{\partial^{3} u_{0}}{\partial t^{2} \partial x}\right|_{x=l-a t}=\left.\frac{\partial^{3} u_{1}}{\partial t^{2} \partial x}\right|_{x=l-a t},\left.\frac{\partial^{3} u_{0}}{\partial t \partial x^{2}}\right|_{x=l-a t}=\left.\frac{\partial^{3} u_{1}}{\partial t \partial x^{2}}\right|_{x=l-a t}, \\
& \left.\frac{\partial^{4} u_{0}}{\partial t^{3} \partial x}\right|_{x=l-a t}=\left.\frac{\partial^{4} u_{1}}{\partial t^{3} \partial x}\right|_{x=l-a t},\left.\frac{\partial^{4} u_{0}}{\partial t^{2} \partial x^{2}}\right|_{x=l-a t}=\left.\frac{\partial^{4} u_{1}}{\partial t^{2} \partial x^{2}}\right|_{x=l-a t},\left.\frac{\partial^{4} u_{0}}{\partial t \partial x^{3}}\right|_{x=l-a t}=\left.\frac{\partial^{4} u_{1}}{\partial t \partial x^{3}}\right|_{x=l-a t} .
\end{aligned}
$$

Выполнение этих соотношений приводит к дополнительным условиям согласования (8). Покажем, как получаются эти условия. Рассмотрим условие непрерывности функций $u_{0}$ и $u_{1}$ на $x+a t=l$ :

$$
\begin{aligned}
\left(u_{0}-u_{1}\right)(t, l-a t)=\varphi_{0}(l)-\nu_{1}(0)+t\left[a \nu_{2}(0)+\varphi_{1}(l)-\nu_{1}^{\prime}(0)-a \varphi_{0}^{\prime}(l)\right]+ \\
+\frac{1}{2} t^{2}\left[-\frac{2 a}{l^{2}}\left(3 a \mu_{1}\left(\frac{l}{a}\right)+a l \mu_{2}\left(\frac{l}{a}\right)-3 a \nu_{1}(0)+2 a l \nu_{2}(0)-l \mu_{1}^{\prime}\left(\frac{l}{a}\right)-2 l \nu_{1}^{\prime}(0)\right)\right]+ \\
+\frac{1}{2} t^{2}\left[\varphi_{2}(l)-2 a \varphi_{1}^{\prime}(l)+a^{2} \varphi_{0}^{\prime \prime}(l)\right]+\frac{1}{6} t^{3}\left[\varphi_{3}(l)-3 a \varphi_{2}^{\prime}(l)+3 a^{2} \varphi_{1}^{\prime \prime}(l)-a^{3} \varphi_{0}^{(3)}(l)\right]+ \\
+\frac{1}{6} t^{3}\left[\frac{6 a^{2}}{l^{3}}\left(2 a \mu_{1}\left(\frac{l}{a}\right)+a l \mu_{2}\left(\frac{l}{a}\right)-2 a \nu_{1}(0)+a l \nu_{2}(0)-l \mu_{1}^{\prime}\left(\frac{l}{a}\right)-l \nu_{1}^{\prime}(0)\right)\right] .
\end{aligned}
$$

Данное соотношение имеет вид $A+B t+C t^{2}+D t^{3}$. Преобразуем его с учетом условий согласования (6)-(7). Это условие непрерывности примет вид

$$
\begin{aligned}
&\left(u_{0}-u_{1}\right)(t, l-a t)= \\
&= \frac{1}{2} t^{2}\left[-\frac{2 a}{l^{2}}\left(3 a \mu_{1}\left(\frac{l}{a}\right)+a l \mu_{2}\left(\frac{l}{a}\right)-3 a \varphi_{0}(l)-2 l \varphi_{1}(l)+2 a l \varphi_{0}^{\prime}(l)-l \mu_{1}^{\prime}\left(\frac{l}{a}\right)\right)\right]+ \\
&+\frac{1}{2} t^{2}\left[\varphi_{2}(l)-2 a \varphi_{1}^{\prime}(l)+a^{2} \varphi_{0}^{\prime \prime}(l)\right]+\frac{1}{6} t^{3}\left[\varphi_{3}(l)-3 a \varphi_{2}^{\prime}(l)+3 a^{2} \varphi_{1}^{\prime \prime}(l)-a^{3} \varphi_{2}(l)\right]+ \\
&+\frac{1}{6} t^{3}\left[\frac{6 a^{2}}{l^{3}}\left(2 a \mu_{1}\left(\frac{l}{a}\right)+a l \mu_{2}\left(\frac{l}{a}\right)-2 a \varphi_{0}(l)-l \varphi_{0}(l)-l \mu_{1}^{\prime}\left(\frac{l}{a}\right)-a l^{2} \varphi_{0}^{\prime}(l)\right)\right] .
\end{aligned}
$$

Далее, учитывая линейную независимость функций $1, t, t^{2}, t^{3}$, потребуем, чтобы коэффициенты при $t$ и $t^{2}$ обращались в нуль. Получаем первые два уравнения системы

$$
\begin{aligned}
& \varphi_{2}(l)-2 a \varphi_{1}^{\prime}(l)+a^{2} \varphi_{0}^{\prime \prime}(l)-\frac{2 a}{l^{2}}\left(3 a \mu_{1}\left(\frac{l}{a}\right)+a l \mu_{2}\left(\frac{l}{a}\right)-3 a \varphi_{0}(l)-2 l \varphi_{1}(l)+2 a l \varphi_{0}^{\prime}(l)-l \mu_{1}^{\prime}\left(\frac{l}{a}\right)\right)=0 \\
& \varphi_{3}(l)-3 a \varphi_{2}^{\prime}(l)+3 a^{2} \varphi_{1}^{\prime \prime}(l)-a^{3} \varphi_{2}(l)+\frac{6 a^{2}}{l^{3}}\left(2 a \mu_{1}\left(\frac{l}{a}\right)+a l \mu_{2}\left(\frac{l}{a}\right)-2 a \varphi_{0}(l)-l \varphi_{1}(l)-l \mu_{1}^{\prime}\left(\frac{l}{a}\right)-\right. \\
& \left.-a l^{2} \varphi_{0}^{\prime}(l)\right)=0 .
\end{aligned}
$$

Аналогично рассматриваются все оставшиеся соотношения. Каждое соотношение записывается в виде полинома третьей степени, учитывая вид решений (9), (10). Затем его коэффициенты упрощаются за счет условий $(6),(7)$, после чего они приравниваются к нулю. Получается система уравнений, которую решаем относительно $\mu_{1}^{(i)}(l / a), i=\overline{0,5}, \mu_{2}^{(i)}(l / a), i=\overline{0,4}, \nu_{1}^{(5)}(0), \nu_{2}^{(4)}(0)$. Ee peшение и есть условия (8). Устойчивость решения $u_{0}$ по $\varphi_{j}, j=0,1,2,3$, в $\overline{Q^{(0)}}$ и $u_{1}$ по $\mu_{i}, \nu_{i}, i=1,2$, в $\overline{Q^{(1)}}$ непосредственно следует из формул (9) и (10). Теорема доказана.

Замечание. В процессе решения смешанной задачи (1)-(4) использовались средства компьютерной алгебры - система Mathematica 11: для проверки решений $u_{0}, u_{1}$ и проверки дополнительных условий согласования (8).

Заключение. В работе построено единственное классическое решение смешанной задачи в полуполосе для линейного нестрого гиперболического однородного уравнения четвертого порядка, оператор которого представляет собой произведение одинаковых операторов первого порядка. Получены достаточные требования гладкости входных данных и достаточные условия согласования граничных условий с начальными условиями и уравнением.

Благодарность. Авторы выражают благодарность доктору физико-математических наук, профессору Ломовцеву Федору Егоровичу за внимательное знакомство с рукописъю статьи и сделанные ценные замечания. 


\section{Список литературы}

1. Иврий Н. И., Петков В. М. 1974. Необходимые условия корректности задачи Коши для нестрого гиперболических уравнений. Успехи матем. наук, 29(5): 3-70.

2. Ломовцев Ф. Е., Юрчук Н. И. 2016. Решение начально-краевой задачи для нестрого гиперболического уравнения при смешанных граничных условиях в четверти плоскости. Весці НАН Б, 3: 51-57.

3. Ломовцев Ф. Е. 2019. Критерий корректности смешанной задачи для одного параболического уравнения на отрезке со смешанными граничными условиями на концах. Материалы Междунар. конференции «Воронежская зимняя математическая школа» (28 февраля - 2 марта 2019 г.) Воронеж: Издательский дом ВГУ: 184-185.

4. Чеб Е. С. 2017. Классическое решение смешанной задачи для линейного гиперболического уравнения четвертого порядка с кратными характеристиками. Вестник ГГУ. Сер. 2, 7(3): 3341.

5. Sakamoto P. 1970. Mixed problems for hyperbolic equations I, II. J. Math. Kyoto Univ., 10(3): $403-417$.

6. Than D. V., Mikio T., Nguen D. S. 2000. The characteristic method and its generalization for firstorder nonlinear partial differential equations, CHAPMAN\&HALL/CRC. Monographs and Surveys in Pare and Applid Mathematics, 101. Boca Raton-London-New York-Washington, 237 p.

\section{References}

1. Ivrii N. I., Petkov V. M. 1974. Neobhodimye usloviya korrektnosti zadachi Koshi dlya nestrigo giperbolicheskih uravneniy [Necessary condition for the Cauchy problem for non-strictly hyperbolic equations]. Uspehi matem. Nauk, 29(5): 3-70.

2. Lomovtsev F. E., Yurchuk N. I. 2016. Reshenie nachalno-kraevoy zadachi dlay nestrogo giperbolicheskogo uravnebiya pri smeshannih granichnih usloviayh v chetverti ploskosti [Initial boundary value problem for non-strictly hyperbolic equation with mixed boundary condition in a quadrant]. Vesti NANB. Ser. fiz.-math.n., 3: 51-57.

3. Lomovtsev F. E. 2019. Kriteriy korrektnosti smeshannoy zadachi dlya odnogo parabolicheskogo uravneniya na otrezke so smeshannimi granichnimi usloviyami na koncah Materialy Mezhd. Konf. «Voronezhskaya zimnya matem shkola»(28.02-02.03 2019). Voronezh, VGU: 184-185.

4. Cheb E. S. 2017. Klassicheskoe reshenie smeshannoy zadachi dlya lineynogo giperbolicheskogo uravneniya chetvertogo poryadka s kratnimi harakteristikami [Classical solution of the mixed problem for linear hyperbolic fourth-order equation with multiple characteristics]. Vestnik GSU. S. 27(3): 33-41.

5. Sakamoto P. 1970. Mixed problems for hyperbolic equations I, II. J. Math. Kyoto Univ., 10(3): 403-417.

6. Than D. V., Mikio T., Nguen D. S. 2000. The characteristic method and its generalization for firstorder nonlinear partial differential equations, CHAPMAN\&HALL/CRC. Monographs and Surveys in Pare and Applid Mathematics, 101. Boca Raton-London-New York-Washington, 237 p.

Симинская Елена Сергеевна - выпускница магистратуры ФПМИ Белорусского государственного университета

пр. Независимости, 4, г. Минск, Республика Беларусь, 220030

E-mail: Slavelena16@gmail.com

Чеб Елена Сергеевна - кандидат физико-математических наук, доцент кафедры компьютерных технологий и систем ФПМИ Белорусского государственного университета

пр. Независимости, 4, г. Минск, Республика Беларусь, 220030

E-mail: cheb@bsu.by 